\title{
Prevalence of Metabolic Syndrome in Patients with Psoriasis
}

\author{
Ilkin Zindanc1, Ozlem Albayrak, Mukaddes Kavala, \\ Emek Kocaturk, Burce Can, Sibel Sudogan, and Melek Koç \\ Department of Dermatology, Goztepe Training and Research Hospital, Istanbul, Turkey \\ Correspondence should be addressed to Ilkin Zindancı, ilkin.dr@gmail.com
}

Received 22 November 2011; Accepted 21 December 2011

Academic Editors: A. Cuocolo and L. Kuller

Copyright ( 2012 Ilkin Zindanc1 et al. This is an open access article distributed under the Creative Commons Attribution License, which permits unrestricted use, distribution, and reproduction in any medium, provided the original work is properly cited.

\begin{abstract}
Background. Psoriasis is a chronic inflammatory skin disorder in which proinflammatory cytokines including IL-6 and TNF- $\alpha$ increase both locally and systematically. It is thought that chronic inflammation results in metabolic diseases and proinflammatory cytokines give rise to the development of atherogenesis, peripheral insulin resistance, hypertension, and type 2 diabetes. Our aim was to investigate the prevalence of metabolic syndrome in patients with psoriasis vulgaris. Methods. Study consisted of 115 plaquetype psoriasis patients and 140 healthy individuals. Data including body weight, height, waist circumference, body-mass index, and arterial blood pressure were collected. Fasting blood glucose, triglyceride, and HDL levels were determined. International Diabetes Federation Criteria for Metabolic Syndrome and Insulin Resistance were used for evaluating patients with metabolic syndrome and diabetes. Results. Compared to the control group, metabolic syndrome, diabetes mellitus, and hypertension were found to be higher in psoriasis patients. Metabolic syndrome was increased by 3 -folds in psoriasis patients and was more prevalent in women than in men. It was determined that the prevalence of metabolic syndrome was higher in psoriasis patients after the age of 40. Metabolic syndrome was not related to smoking, severity of psoriasis, and duration of disease. Conclusions. Our findings suggest that psoriasis preconditions occurrence of a group of diseases such as diabetes mellitus, hypertension, and metabolic syndrome. For this reason, patients with psoriasis should be treated early and they should be followed with respect to metabolic diseases.
\end{abstract}

\section{Introduction}

Psoriasis is a chronic inflammatory skin disorder which is characterized by local and systemic escalation of proinflammatory cytokines such as IL- 6 and TNF- $\alpha[1,2]$. Proinflammatory cytokines which take place in chronic inflammation are accused of augmentation of atherogenesis and peripheric insulin resistance and thus leading to hypertension and type II Diabetes Mellitus (DM) [2,3]. Recent research reported an association between psoriasis and metabolic disorders such as obesity, dyslipidemia, and type II DM and it is shown that severe psoriasis might be associated with increased mortality rate due to cardiovascular disorders $[2,4-6]$. Since psoriasis is characterized by a systemic inflammation which involves numerous cytokines and inflammatory markers in particular TNF- $\alpha$, it may predispose the patients to metabolic disorders. We aimed to investigate the prevalence of metabolic syndrome in patients with psoriasis vulgaris.

\section{Patients and Methods}

The study included 115 psoriasis vulgaris patients and 140 healthy, sex, and age-matched control subjects who do not have psoriasis and any systemic disease. Inclusion criteria for cases were age more than 18 years, clinical diagnosis of chronic plaque psoriasis (lasting at least 6 months), and not receiving any systemic or local antipsoriatic treatment for the last 4 weeks. Informed consent was given by all the patients who enrolled in the study. Information sheets for the patients included age, gender, weight, height, body mass index (BMI), waist circumference, smoking habits, blood pressure, age of onset, and duration of the disease. Exclusion criteria were pregnancy and psoriatic arthritis. Severity of psoriasis was assessed by psoriasis area severity index (PASI) and accepted as severe in patients with PASI $>10$ and mild/moderate in patients with PASI $\leq 10$ [7]. BMI was calculated as weight/height $\left(\mathrm{kg} / \mathrm{m}^{2}\right)$ and metabolic 
syndrome was diagnosed in the presence of central obesity in addition to two or more criteria of the International Diabetes Foundation: waist circumference $\geq 94 \mathrm{~cm}$ in men or $\geq 80 \mathrm{~cm}$ in women; hypertriglyceridemia $\geq 150 \mathrm{mg} / \mathrm{dL}$; HDL $<40 \mathrm{mg} / \mathrm{dL}$ in men or $<50 \mathrm{mg} / \mathrm{dL}$ in women; blood pressure $\geq 130 / 85 \mathrm{mmHg}$; fasting blood glucose $\geq 100 \mathrm{mg} / \mathrm{dL}$ [8]. Triglyceride and HDL levels were calculated by enzymatic assay, glucose was calculated by glucose oxidase assay. Student's $t$-test, Mann-Whitney $U$, chi-square, and Fisher's exact chi-square tests were used for statistical analysis of the data. Enter logistical regression analysis was used for multivariate determination of parameters affecting metabolic syndrome. $P$ values $<0.05$ were accepted as statistically significant and confidence interval (CI) was $95 \%$.

\section{Results}

The study group included $64(55.7 \%)$ females and 51 (44.3\%) males with a mean age of 45.4 (range 19-79) years. The control group consisted of $70(50 \%)$ females and 70 (50\%) males with a mean age of 43.4 (range 19-70) years. Duration of the disease was $11.4 \pm 9.2$ years, and mean age of onset were $34.24 \pm 17.3$ years. Severity of psoriasis was mild to moderate in $76(66.1 \%)$ of the patients and severe in $39(33.9 \%)$ of the patients with a mean PASI of $9.98 \pm 8.6$. There was a significant difference between the smoking rates of the groups which were $35.7 \%$ and $24.3 \%$ in psoriasis and control groups, respectively $(P<0.05)$. Waist circumference $\geq 94 \mathrm{~cm}$ in men and $\geq 80 \mathrm{~cm}$ in women was observed in $73 \%$ and $83.6 \%$ of psoriasis and control subjects, respectively; waist circumference was significantly slimmer in psoriasis patients $(P<0.05)$. Mean BMI was $28.73 \pm 6.12 \mathrm{~kg} / \mathrm{m}^{2}$ and $28.22 \pm 4,9 \mathrm{~kg} / \mathrm{m}^{2}$ in psoriasis and control groups, respectively. No significant differences were found between groups with respect to BMI $(P>0.05)$. There were also no significant differences between groups with respect to high triglyceride levels and low HDL levels $(P>0.05)$. In contrast, we found a significant correlation between psoriasis and hypertension, diabetes mellitus and metabolic syndrome. Hypertension and diabetes mellitus were significantly more common in psoriasis patients as compared to controls. We found a higher prevalence of MS in psoriasis patients than in controls (53\% versus 39\%) $(P<$ $0.01)$. The prevalence of various components of MS in cases and controls along with $P$ values is given in Table 1 .

Although all patients with MS had waist circumference $\geq 80 \mathrm{~cm}$ in females and $\geq 94 \mathrm{~cm}$ in males, there was no significant difference with respect to mean BMI between psoriasis patients and control cases with MS $(P>0.05)$. But we observed that female psoriasis patients with MS had a higher BMI $(30.60 \pm 7.18$ in females versus $26.37 \pm 3.37$ in males $)(P<0.01)$.

Considering the data for diabetes mellitus, hypertension, and MS in psoriasis patients, it appeared that deviations from normal were most prevalent at the fifth decade of life (Table 2). No apparent differences were noted regarding the prevalence of DM and hypertension between the genders $(P>0.05)$. In contrast, we found a significant correlation between MS and female gender. MS was more common in female patients than male patients $(P<0.05)$. The mean age of psoriasis onset in patients with MS was significantly higher than patients without MS $(P<0.01)$. Prevalence of MS was not correlated to smoking, severity of psoriasis, and duration of disease $(P>0.05)$ (Table 3$)$.

Multiple logistic regression analysis revealed that MS is increased by 2.94 -fold in psoriasis patients (95\% CI : $1.40-$ 6.19). When the parameters that might influence MS in psoriasis patients such as age, gender, age of onset, and smoking were evaluated, the model was found highly significant $(P<0.001)$ with a Negelkerke $\left(R^{2}\right)$ value of 0.293 and determination coefficient of $72.2 \%$. The influence of female gender and age on the occurrence of MS in psoriasis patients was found significant $(P<0.01)$. Female gender increased the risk of MS by 3.195-fold (95\% CI : 1.12-9.04) compared to males; patients aged between $40-49$ had an increased risk of MS by 5.141 -fold (95\% CI : 1.75-24.47), patients aged over 60 had an increased risk of MS by 6.531 -fold (95\% CI : 1.553.37). It was found that MS was independent from smoking habit (Table 4).

\section{Discussion}

This study reveals that hypertension, diabetes mellitus, and MS are significantly more common in psoriasis patients than controls. Therefore, all dermatologists should be aware of MS or individual components of MS might be associated with psoriasis during the course of this disease. Recent studies showed that psoriasis is associated with metabolic disorders such as hypertension, type II DM, dyslipidemia, abdominal obesity, insulin resistance, and cardiac disorders and the risk of metabolic syndrome is increased in patients with psoriasis [2-6, 9-12]. Sommer et al. reported that there is a significant association between psoriasis and type II DM, hypertension, hyperlipidemia, and coronary artery disease and MS is increased by twofolds in a study they conducted in 581 patients [3]. Other studies showed an increased frequency of ischemic heart disease, DM, hypertension, and dyslipidemia in patients with psoriasis when compared to controls $[13,14]$. Gisondi et al. found increased prevalence of hypertrygliceridemia and MS in psoriasis patients compared to controls, but they did not find any difference between psoriasis patients and controls with respect to low levels of HDL, DM, and hypertension [15]. Farshchian et al. failed to demonstrate any difference between psoriasis patients and controls with regard to fasting blood glucose, triglyceride, cholesterol, HDL, LDL, and VLDL levels [16]. In our study we observed that psoriasis is associated with smoking, DM, hypertension, and MS. DM and hypertension was accompanying our psoriasis patients along with MS. These findings confirmed the literature $[3,11,13,14]$. It is reported that smoking is more prevalent in psoriasis patients [13-15]. We found the rates of smoking higher than controls. Levels of triglyceride and cholesterol were reported to be high in psoriasis patients $[3,13,14]$. We did not detect any dyslipidemia in our patients. This was consistent with previous findings which found normal lipid levels in 
TABLE 1: Metabolic syndrome criteria in psoriasis patients and controls.

\begin{tabular}{|c|c|c|c|c|}
\hline & & $\begin{array}{c}\text { Psoriasis }(n=115) \\
n(\%)\end{array}$ & $\begin{array}{c}\text { Control }(n=140) \\
n(\%)\end{array}$ & ${ }^{++} P$ \\
\hline \multirow[t]{2}{*}{ Waist circumference $(\mathrm{cm})$} & $\mathrm{M} \geq 94 ; \mathrm{F} \geq 80$ & $84(73.0 \%)$ & $117(83.6 \%)$ & \multirow[t]{2}{*}{$0,041^{*}$} \\
\hline & $\mathrm{M}<94 ; \mathrm{F}<80$ & $31(27.0 \%)$ & $23(16.4 \%)$ & \\
\hline $\operatorname{BMI}\left(\mathrm{kg} / \mathrm{m}^{2}\right)$ & & $28.73 \pm 6.1$ & $28.22 \pm 4.9$ & 0.473 \\
\hline \multirow[t]{2}{*}{ Triglyceride (mg/dL) } & $\geq 150$ & $52(45.2 \%)$ & $55(39.3 \%)$ & \multirow[t]{2}{*}{0.340} \\
\hline & $<150$ & $63(54.8 \%)$ & $85(60.7 \%)$ & \\
\hline \multirow[t]{2}{*}{$\mathrm{HDL}(\mathrm{mg} / \mathrm{dL})$} & $\mathrm{F} \leq 50 ; \mathrm{M} \leq 40$ & $50(43.5 \%)$ & $46(32.9 \%)$ & \multirow[t]{2}{*}{0.082} \\
\hline & $\mathrm{F}>50 ; \mathrm{M}>50$ & $65(56.5 \%)$ & $94(67.1 \%)$ & \\
\hline \multirow[t]{2}{*}{$\mathrm{BP}(\mathrm{mmHg})$} & $\geq 130 / 85$ & $47(40.9 \%)$ & $33(23.6 \%)$ & \multirow[t]{2}{*}{$0.003^{* *}$} \\
\hline & $<130 / 85$ & $68(59.1 \%)$ & $107(76.4 \%)$ & \\
\hline \multirow[t]{2}{*}{$\mathrm{FBG}(\mathrm{mg} / \mathrm{dL})$} & $\geq 100$ & $56(48.7 \%)$ & $49(35.0 \%)$ & \multirow[t]{2}{*}{$0.027^{*}$} \\
\hline & $<100$ & $59(51.3 \%)$ & $91(65.0 \%)$ & \\
\hline \multirow[t]{2}{*}{ MS } & $(+)$ & $61(53.0 \%)$ & $59(39.0 \%)$ & \multirow[t]{2}{*}{$0.004^{* *}$} \\
\hline & $(-)$ & $54(47.0 \%)$ & $91(65.0 \%)$ & \\
\hline
\end{tabular}

${ }^{++}$Chi-square test, ${ }^{*} P<0.05,{ }^{* *} P<0.01$.

BMI: Body Mass Index; MS: Metabolic syndrome; F: Female; M: Male; BP: Blood pressure; FBG: Fasting blood glucose.

TABLE 2: Association of MS, DM, and HT with age in psoriasis patients.

\begin{tabular}{|c|c|c|c|c|c|c|}
\hline & & \multirow{2}{*}{$n$} & \multicolumn{3}{|c|}{ Age } & \multirow[t]{2}{*}{$P$} \\
\hline & & & Min-max & Med & $\mathrm{SD}$ & \\
\hline \multirow[t]{2}{*}{ MS } & & 59 & $18-79$ & 51.28 & 14.43 & \multirow[t]{2}{*}{$0.001^{* *}$} \\
\hline & $(-)$ & 56 & $17-79$ & 39.16 & 14.05 & \\
\hline \multirow[t]{2}{*}{$\mathrm{DM}$} & $(+)$ & 56 & $23-79$ & 52.62 & 14.13 & \multirow[t]{2}{*}{$0.001^{* *}$} \\
\hline & $(-)$ & 59 & $17-77$ & 38.50 & 13.44 & \\
\hline \multirow[t]{2}{*}{$\mathrm{HT}$} & $(+)$ & 47 & $27-79$ & 54.76 & 13.07 & \multirow[t]{2}{*}{$0.001^{* *}$} \\
\hline & $(-)$ & 68 & $17-71$ & 38.89 & 15.55 & \\
\hline
\end{tabular}

+Student's $t$-test, $* * P<0.01$.

psoriasis patients and concluded that hyperlipidemia did not have a clinical significance in psoriasis patients $[15,16]$. It has been reported that hypertension and DM were common in psoriasis patients regardless of gender and hypertension had an increased frequency by advanced age while DM might be seen in any age $[3,13,14]$. In our study, we found both hypertension and DM in advanced age, regardless of gender.

Although obesity is reported more frequent among psoriasis patients, we have not found BMI and waist circumference significantly higher in psoriasis patients $[3,13$, 14]. However we observed a higher prevalence of MS among psoriasis patients than controls. This may be explained by the literature which concluded that the association between psoriasis and MS is independent from the tendency of psoriatic patients to be obese [15].
In our study we observed that MS was increased by 3 -fold in psoriasis patients. The association of psoriasis with MS was independent from smoking. These findings confirmed those in the literature $[11,13,14,17]$. We found the prevalence of MS increased by 5 -fold in patients aged between 40 and 59 years. This was consistent with previous studies by Sommer et al. and Gisondi et al. who reported that MS was significantly higher in psoriasis patients after the age of $40[3,15]$. Cohen et al. also demonstrated that the association between psoriasis and MS was pronounced after the age of 50 [13]. However Nisa and Qazi observed the higher prevalence of MS in psoriasis patients in the age group $18-30$ [17].

In contrast to the reports that MS was regardless of gender, we observed a significant relationship between MS 
TABLE 3: The associations of MS with patient characteristics in psoriasis patients.

\begin{tabular}{|c|c|c|c|c|}
\hline \multirow{3}{*}{ Psoriasis group } & & \multicolumn{2}{|c|}{ MS } & \multirow{3}{*}{$P$} \\
\hline & & $(+)$ & $(-)$ & \\
\hline & & $\mathrm{M} \pm \mathrm{SD}($ Median $)$ & $\mathrm{M} \pm \mathrm{SD}($ Median $)$ & \\
\hline${ }^{+}$Duration of disease & & $12.29 \pm 9.65$ & $10.42 \pm 8.55$ & 0.277 \\
\hline \multirow[t]{2}{*}{${ }^{+}$Age of onset } & & $39.73 \pm 17.03$ & $28.03 \pm 15.56$ & $0.001^{* *}$ \\
\hline & & $n(\%)$ & $n(\%)$ & ${ }^{++} P$ \\
\hline \multirow[t]{4}{*}{ Gender } & Male & $21(41.2 \%)$ & $24(58.8 \%)$ & $0.023^{*}$ \\
\hline & Female & $40(62.5 \%)$ & $30(37.5 \%)$ & \\
\hline & No & $28(54.9 \%)$ & $23(45.1 \%)$ & \\
\hline & Ex-smoker & $12(66.7 \%)$ & $6(33.3 \%)$ & 0.166 \\
\hline \multirow[t]{2}{*}{ Smoking } & Active smoker & $17(41.4 \%)$ & $24(58.6 \%)$ & \\
\hline & Passive smoker & $4(80.0 \%)$ & $1(20.0 \%)$ & \\
\hline \multirow[t]{2}{*}{ PASI } & Mild-moderate & $39(51.3 \%)$ & $37(48.7 \%)$ & 0.604 \\
\hline & Severe & $22(56.4 \%)$ & $17(43.6 \%)$ & \\
\hline
\end{tabular}

${ }^{+}$Student's $t$-test, ${ }^{++}$Chi-square test, ${ }^{*} P<0.05,{ }^{* *} P<0.01$.

TABLE 4: Multiple regression analysis.

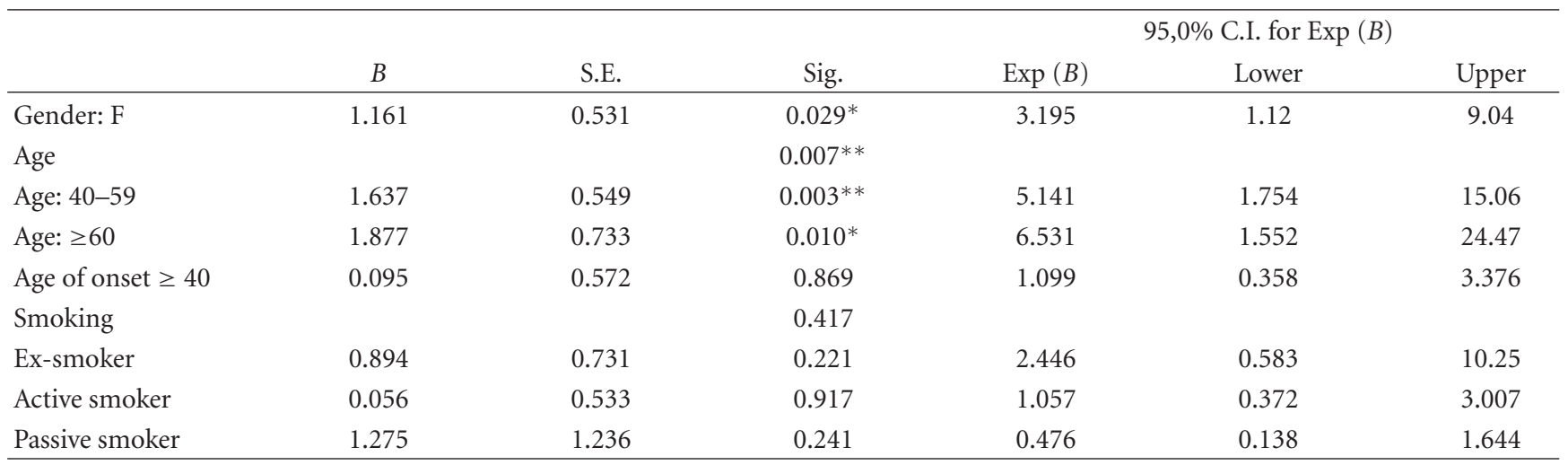

and gender $[3,11,15,17]$. MS was more prevalent in women and female gender increased the prevalence of MS by 3 -fold.

High BMI levels were frequent in our female patients with MS and this finding was confirming the previous findings [3]. We believe that high frequency of MS in female patients is the result of increased waist circumference due to high BMI.

It was reported that MS is related to the duration of the disease, psoriasis starts in young ages in patients with MS and duration of the disease is longer in patients with MS $[3,15]$. But we observed that psoriasis started at advanced age in our patients with MS and MS was not related to the duration of the disease. There are controversies in the literature about the association of MS with severity of the disease. Sommer et al. reported a positive relation with severity, while Gisondi et al. and Nisa and Qazi declared an independent relation [3, 15, 17]. Like Gisondi et al. and Nisa and Qazi, we did not observe an association between severity of the disease and MS.

Our results showed that psoriasis predisposes to the development of DM, hypertension, and MS and psoriasis increased the prevalence of MS by 3-fold. Therefore, we recommend evaluating psoriasis patients for the presence of metabolic diseases which may interfere with the patients' health for the rest of their living.

\section{References}

[1] M. P. Schon and W. H. Boehncke, "Psoriasis," New England Journal of Medicine, vol. 352, no. 18, pp. 1899-1912, 2005.

[2] T. Henseler and E. Christophers, "Disease concomitance in psoriasis," Journal of the American Academy of Dermatology, vol. 32, no. 6, pp. 982-986, 1995.

[3] D. M. Sommer, S. Jenisch, M. Suchan, E. Christophers, and M. Weichenthal, "Increased prevalence of the metabolic syndrome in patients with moderate to severe psoriasis," Archives of Dermatological Research, vol. 298, no. 7, pp. 321-328, 2006.

[4] L. Mallbris, C. T. Ritchlin, and M. Ståhle, "Metabolic disorders in patients with psoriasis and psoriatic arthritis," Current Rheumatology Reports, vol. 8, no. 5, pp. 355-363, 2006.

[5] L. Mallbris, O. Akre, F. Granath et al., "Increased risk for cardiovascular mortality in psoriasis inpatients but not in outpatients," European Journal of Epidemiology, vol. 19, no. 3, pp. 225-230, 2004.

[6] J. M. Gelfand, A. L. Neimann, D. B. Shin, X. Wang, D. J. Margolis, and A. B. Troxel, "Risk of myocardial infarction in patients with psoriasis," Journal of the American Medical Association, vol. 296, no. 14, pp. 1735-1741, 2006.

[7] A. Y. Finlay, "Current severe psoriasis and the rule of tens," British Journal of Dermatology, vol. 152, no. 5, pp. 861-867, 2005. 
[8] I. S. Prodromas, S. Stylionos, and T. Dimitros, "Metabolic syndrome in rheumatic diseases: epidemiology, pathophysiology, and clinical implications," Arthritis Research and Therapy, vol. 10, no. 3, p. 207, 2008.

[9] E. Christophers, "Comorbidities in psoriasis," Clinics in Dermatology, vol. 25, no. 6, pp. 529-534, 2007.

[10] M. Wakkee, H. B. Thio, E. P. Prens, E. J. G. Sijbrands, and H. A. M. Neumann, "Unfavorable cardiovascular risk profiles in untreated and treated psoriasis patients," Atherosclerosis, vol. 190, no. 1, pp. 1-9, 2007.

[11] M. Malerba, P. Gisondi, A. Radaeli, R. Sala, P. G. Calzavara Pinton, and G. Girolomoni, "Plasma homocysteine and folate levels in patients with chronic plaque psoriasis," British Journal of Dermatology, vol. 155, no. 6, pp. 1165-1169, 2006.

[12] J. Shapiro, A. D. Cohen, M. David et al., "The association between psoriasis, diabetes mellitus, and atherosclerosis in Israel: a case-control study," Journal of the American Academy of Dermatology, vol. 56, no. 4, pp. 629-634, 2007.

[13] A. D. Cohen, M. Sherf, L. Vidavsky, D. A. Vardy, J. Shapiro, and J. Meyerovitch, "Association between psoriasis and the metabolic syndrome: a cross-sectional study," Dermatology, vol. 216, no. 2, pp. 152-155, 2008.

[14] S. A. Rahat and J. M. Gelfand, "Psoriasis and metabolic disease: epidemiology and pathophysiology," Current Opinion in Rheumatology, vol. 20, no. 4, pp. 416-422, 2008.

[15] P. Gisondi, G. Tessari, A. Conti et al., "Prevalence of metabolic syndrome in patients with psoriasis: a hospital-based casecontrol study," British Journal of Dermatology, vol. 157, no. 1, pp. 68-73, 2007.

[16] M. Farshchian, A. Zamanian, M. Farshchian, A. R. Monsef, and H. Mahjub, "Serum lipid level in Iranian patients with psoriasis," Journal of the European Academy of Dermatology and Venereology, vol. 21, no. 6, pp. 802-805, 2007.

[17] N. Nisa and M. Qazi, "Prevalence of metabolic syndrome in patients with psoriasis," Indian Journal of Dermatology, Venereology and Leprology, vol. 76, no. 6, pp. 662-665, 2010. 


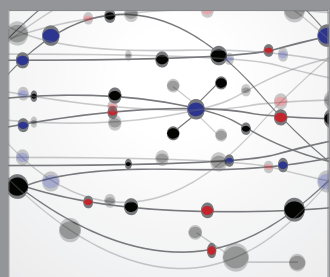

The Scientific World Journal
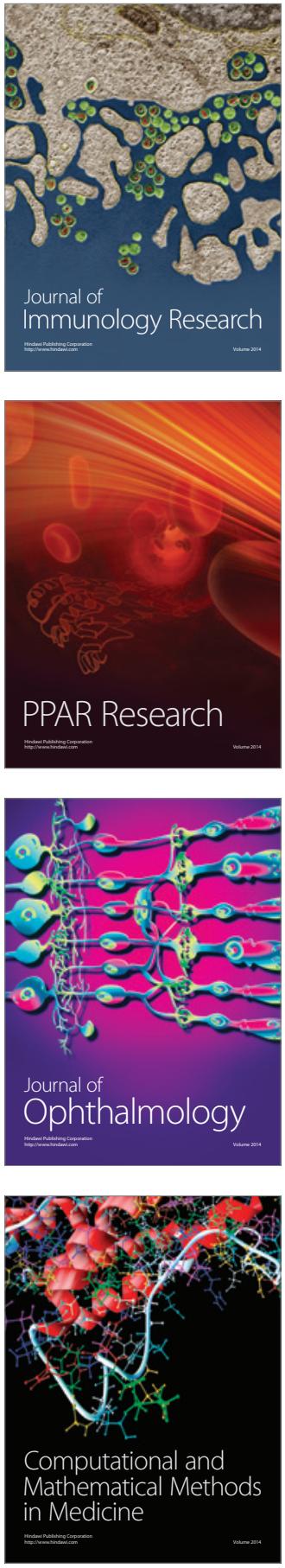

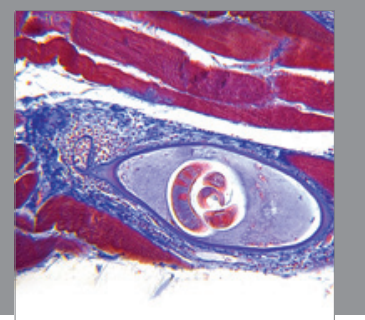

Gastroenterology

Research and Practice
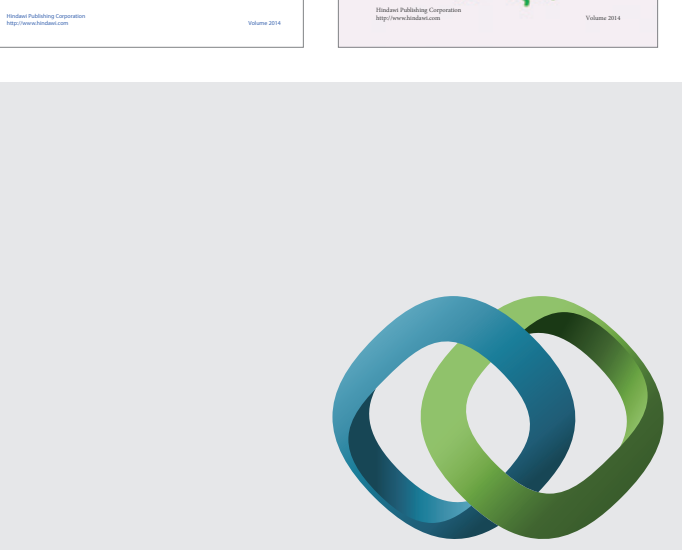

\section{Hindawi}

Submit your manuscripts at

http://www.hindawi.com
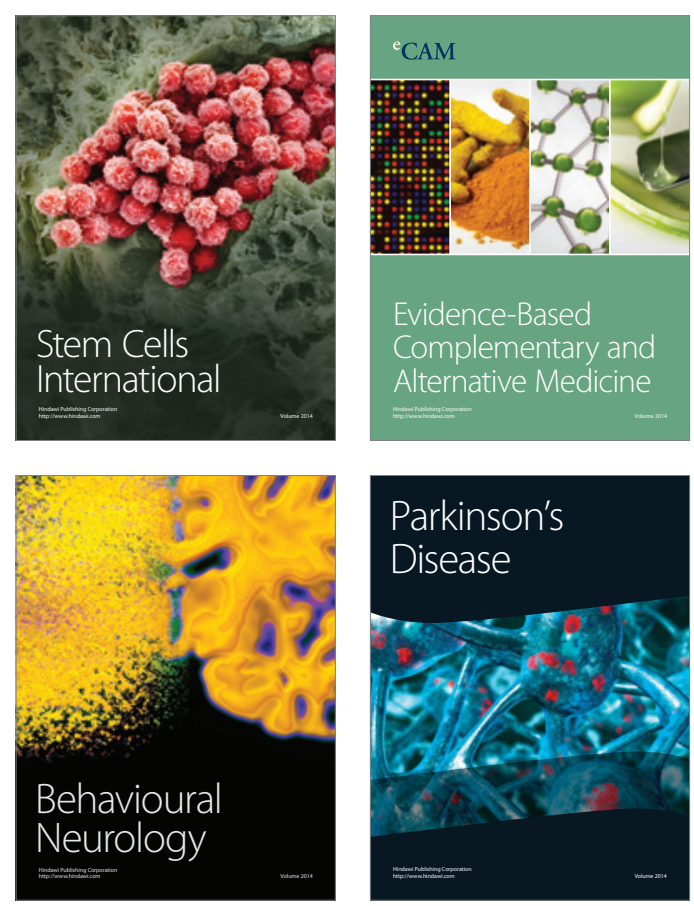

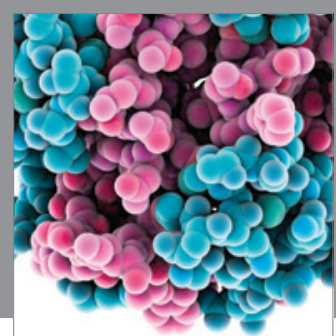

Journal of
Diabetes Research

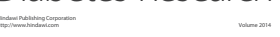

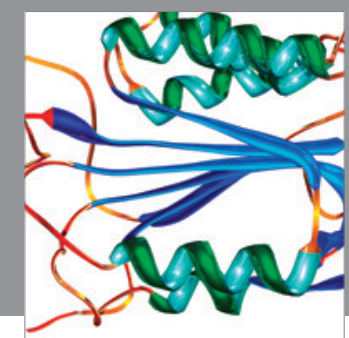

Disease Markers
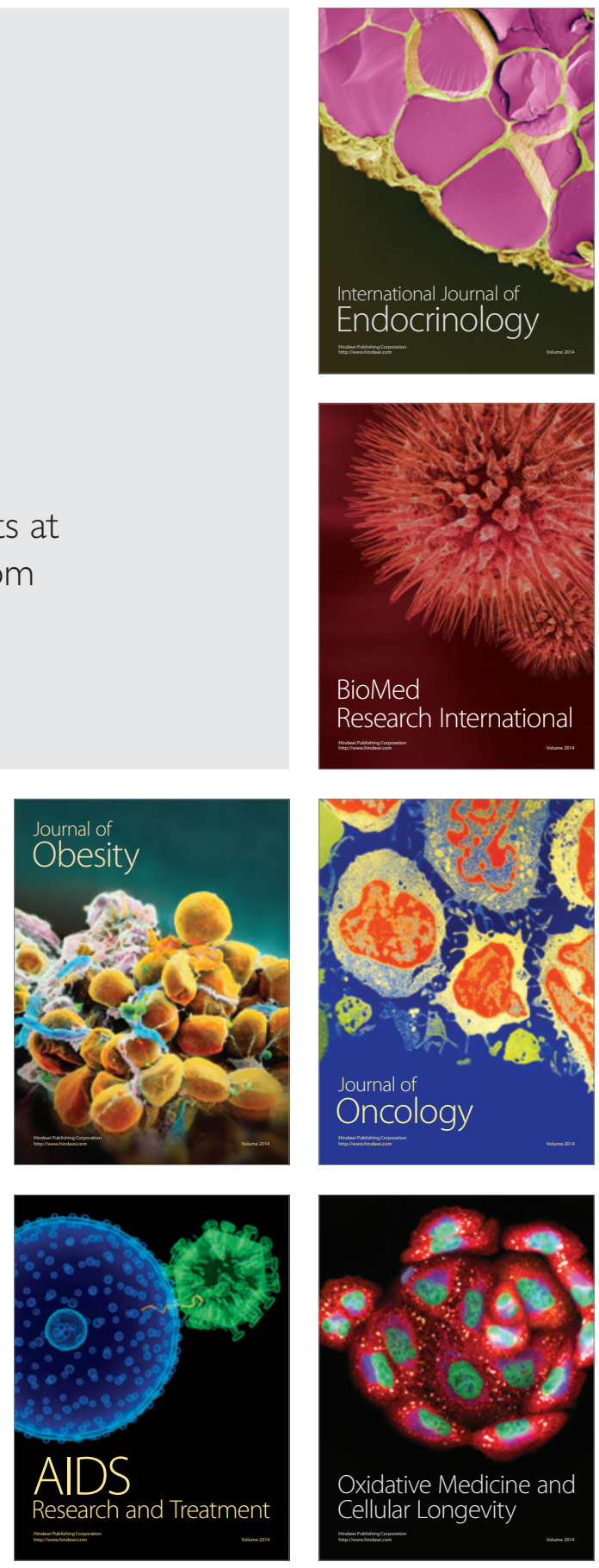